\title{
Green Cloud Computing: Some Studies on Energy Usage Reduction Methods
}

\author{
V. Venkata Sai Lakshmi and N.Thirupathi Rao \\ Dept. of Computer Science \& Engineering, \\ Vignan's Institute of Information Technology, Visakhapatnam, AP, India
}

\begin{abstract}
Distributed computing center around the information figuring proficiency where as green distributed computing is another reasoning which depends on distributed computing design and concentrates on the vitality productivity of gadget and processing. Green Cloud Computing is an approach used to enhance the usage of figuring assets those are being utilized as a part of distributed computing system, for example, stockpiling, servers, its application, and benefits and decrease vitality utilization of these assets which enhances control proficiency. This is finished by different innovations, for example, virtualization and virtual machines movement. This paper surveys the different systems purposed by the distinctive creators to make distributed computing more vitality proficient. The primary target of this paper is to think about and break down the idea of vitality proficient server farm engineering, asset allotment and streamlining.
\end{abstract}

Keywords: Cloud computing, Energy efficiency, Virtualization

\section{Introduction}

Distributed computing is relevant virtualization idea for productive utilization of equipment and programming. It intends to furnish straightforwardness to its end clients with the assistance of on request self-benefit, wide system get to, asset pooling, fast adaptability and estimated execution [1][2]. In distributed computing assets (equipment or programming) are made accessible by at least one supplier. These assets can be utilized by various clients on the paid premise. These administrations gave by distributed computing are extensively groups for example, Amazon, Sales drive, Microsoft, Google, IBM and Sun-Microsystems has set up numerous new server farms for facilitating cloud applications, business application, gaming entryway, media content conveyance and logical preparing. For running these server farms an enormous measure of vitality is required [3]. Power is required for screens, comfort, arrange peripherals, cooling devotees of processor and cooling framework [4]. In spite of the fact that distributed computing give money related advantages however its influence utilization and carbon outflow has turned into a noteworthy natural concern. Server farms store substantial measure of information in cloud and vast measure of vitality is squandered as warmth on cloud. Cloud has turned out to be one of the real sources to an unnatural weather change in view of numerous an Earth-wide temperature boost gases like carbon dioxide, carbon monoxide and so on are discharged amid the power age process.

Green figuring is characterized as a domain maintainable processing. Green processing productively deals with its assets by keeping condition at focus. The fundamental goal of green figuring is to build the vitality productivity and lessen $\mathrm{CO} 2$ discharge. The analysts

Article history:

Received (February 19, 2018), Review Result (March 21, 2018), Accepted (April 18, 2018) 
have given an assortment of programming and equipment answers for the issue of vitality proficiency in cloud operation by limiting the effect of distributed computing on the earth. Virtualization innovation can be utilized to show signs of improvement asset separation and less vitality utilization through live movement and combination. The analysts recommend three sorts of answers for making distributed computing green distributed computing that is condition neighborly. To begin with arrangements programming enhancement, second equipment streamlining and third is organize improvement. Assist there are two programming approaches that are utilized for decreasing vitality utilization: lessening the vitality devoured by memory and diminishing the vitality devoured by memory.

\subsection{Power saving approaches}

Vitality utilization and execution of the framework rely upon numerous elements. Some straightforward procedures give fundamental vitality administration to servers in Cloud situations. Different systems for sparing vitality incorporate utilization of Dynamic Voltage/Frequency Scaling (DVFS) [5][6][7] and utilization of virtualization strategies for better asset use. Different specialists have put numerous endeavors to decrease the vitality utilization in mists and server farms. In this part we display most recent research endeavors by specialist that endeavor to manage them. One of the methods that are as a rule ordinarily utilized as a part of cloud condition is virtualization. The task of numerous VMs helps in solidifying the errand and killing other physical machines by bringing down the utilization of vitality. Live movement alludes to moving the virtual machines starting with one physical server then onto the next straightforwardly. The relocation of virtual machines is observed to be a valuable procedure for making the frameworks more vitality productive. VMM should be possible by utilizing distinctive calculation like first fit, Monte Carlo, Round Robin and so forth. The significant system being utilized as a part of virtual machine relocation is Pre-Copy. As of late, fundamental web firms have influenced gigantic information to focus to enable their online business. Before, processing power essentially centered around centralized servers set a way out of sight since there were no choices aside from a substantial room sized box that could incorporate each vital measure of computational power. Also, the use of switches is as the take after: $17 \%$ assigned for center switches, half for get to switches and 34\% for accumulation switches. Besides, $15 \%$ of aggregate power use is utilized by center and total switches together. Coincidentally, as indicated by the necessities for arrange execution, correspondence quality and load adjusting, the discernible alternative to pick is to save conglomeration and center switches in a disseminated way. The server farm arrange considers for the distinctions among control utilization levels of different server farm outlines. With respect to the 2-Tier arrange, the 3 -Tier organize includes around $25 \mathrm{~kW}$ for collection layer that enable the server farm scale more than 10000 hubs. The 3-Tire with fast (3Ths) organize incorporates less center and conglomeration switches. In addition, the openness of $100 \mathrm{G}$ joins includes some significant downfalls of the augmentation per-switch control use. It is reasoned that a 3Tier system expends under 3Ths system [8]. Figure 1 speaks to a normal circulation of vitality utilization in a 3T server farm. This paper inspected numerous techniques for vitality sparing in server farms for distributed computing and it talks about different methodologies proposed in past research works in this field.

\subsection{Vitality economy resolutions for network}

System foundation is the following principle vitality shopper in server farms. Around $30 \%$ of the whole vitality utilization which used for calculation is devoured by server 
farm arrange [9]. System in Data Centers incorporates switches and interfaces. Chabarek et al. [10] communicated in a paper that the usage of a connection isn't straightforwardly adjusted with its energy utilization. The creators expressed that power utilization relies upon limit of the connection rather than its use. In addition, scientists delineated that vitality utilization of switches depends on an advertiser and it is coordinated with the quantity of line cards and ports included. It ought to be viewed as that power utilization of the two connections and switches are vital for diminishing force utilization by arrange. The four after arrangements are existed for sparing vitality in server farm systems:

Versatile Link Rate strategy is broadly considered on wired systems [11]. Notwithstanding, it has not been utilized on server farm arranges yet. This approach depends on the possibility that vitality utilization of a connection might be diminished its information rate, while movement heap of system joins are low much of the time. In such cases, Adaptive Link Rate diminishes connect vitality use by progressively setting join information rate to its use, while rest mode strategy diminishes vitality use through turning off system assets or putting them to rest mode. In addition, other dynamic system assets should meet QoS prerequisites. Virtual system implanting is the following methodology which is valuable for lessening vitality utilization of system [12]. VNE is for the most part helpful while the system activity is low. The points of system virtualization is to utilize inserting calculations to dole out virtual system assets on a less number of physical framework with an ideal approach [13]. The sit out of gear organize assets could be turned off or put into rest mode.

As of late, Energy mindful steering or green directing for diminishing vitality utilization in server farm arrange is considered [14]. The fundamental thought of Energy mindful directing is to convey steering administration to less number of system assets to decrease vitality use, while supporting system execution. As of late, blended methodologies for sparing vitality in server farms are proposed widely. Mahadevan et al. [15] expressed that lone $16 \%$ of energy utilization might be spared by affecting system vitality diminishing approaches alone. It merits specifying that by consolidating server and system vitality mindful techniques, vitality could be set aside $75 \%$ of vitality use in server farms. By joining vitality sparing methodology, arrange movement union and server workload combination are helpfully utilized. Mahadevan et al. [16] recommends three strategies for sparing vitality in server farms:

NTC diminishes vitality use extensively by evacuating all repetition in the system. This approach solidifies movement on few quantities of connections and switches, and sit without moving connections and switches are deactivated. SLC is an approach to merge organizes activity in a less connections and changes to enable the controller to kill unused assets. To accomplish this objective, SLC exchanges employments to few quantities of servers to kill unused servers. The specialists assessed these three systems on a genuine Web 2.0 contextual investigation in a genuine server farm. The outcomes show that $16 \%$ of vitality could be put away by executing just a connection state adjustment approach, $75 \%$ of vitality could be put away by actualizing both server stack combination and system activity solidification together.

\subsection{Power economy by means of renewable power basis}

Nowadays, stresses over expanding vitality utilization have coordinated to social advantages in controlling the vitality use. Accomplished arrangements comprise of the joining of sustainable power source server farm [17], Yahoo's New York server farm [18], Google server farms, and Microsoft server farms. A sorption chiller influences the 
cooling framework to work by utilizing the warm vitality which recouped from the server farm parts and supplemental sun oriented vitality [3]. It is prescribed to utilize additional vitality, if accessible, from elective recovery frameworks, mostly the sun based vitality, while losing the rest of the vitality from the server farms isn't sufficient to switch on the cooling frameworks. To accomplish a similar reason, the geothermal power and distinctive elective vitality sources can be utilized. To get the cooling unit while the warmth vitality assimilated from the servers isn't sufficient, a two-overlap framework is offered to warm the wet necessary for the ingestion chiller, utilizing sun powered power designing or additional obtainable reestablishing heat source [3].

\section{Existing power competent methods for concentrating on server}

Vitality mindful errand union (ETC) approach that declines vitality utilizes was exhibited by the creators of [3]. Binding the CPU use under a positive beginning and additionally joining capacities inside virtual groups was the purposes for the capacity of ETC in doing as such. Also, this vitality devouring structure perceives the system defer when a capacity moves to another virtual group. Hsu et al. [3] present a method for keeping up the utilization of CPU and overseeing undertaking solidification inside virtual bunches. The undertaking solidification system applies the most suitable method for utilizing assets advantageously. Thus, they tend to contrast the results and the most recent consideration retaining technique, Max Util, which tries to diminish vitality utilization through dedicating the greatest number of conceivable undertakings as it can figure out how to a VM. The outcomes demonstrate that in a cloud framework ETC can vitally limit control misfortune [8]. Along these lines, a need rises for the ETC way to deal with focus on the lower edge of CPU utilize that goes for diminishing loss of vitality through futile servers. Nathuji and Schwan prescribed a virtual power strategy that coordinated 'delicate' and 'hard' scaling systems and joined errand solidification and power administration. The scientists found that this mix may spare more vitality agreeing to the limited number of equipment scaling cases. This technique had a broad virtualization guideline to supply productive methodologies of energy administration.

Raghavendra et al. [3] have considered the issue of energy administration for a server farm condition through blending and orchestrating five diverse power administration methodologies in view of 180 server follows from nine distinctive true undertakings. The specialists researched the issue with respect to control hypothesis and applied a circle to control the criticisms for organizing the controllers' action. These techniques bargains just with the CPU administration and are free of the workload compose. For limiting the vitality utilization, Srikantaiah et al. [4] directed an investigation and inspected the issue of applying the calendar of multi-layered mesh submission in frameworks with varied virtualization. This exploration assessed vitality utilization, execution changes, and asset helpfulness as various workloads with various asset uses are joined on normal servers. Analysts have prescribed an inventive technique for the multidimensional canister pressing issue like a calculation that unites the workloads, to control the proficiency over various assets.

Utilizing DVFS, Wang et al. [5] expand booking calculation which diminishes use of vitality of parallel errand organization. Keeping in mind the end goal to achieve work organization they present a few planning calculations conveying the nature of energy mindful. Concerning actuality that the execution time of irrelevant errands can be produced, the said calculations make the decrease of the supply voltages of CPUs conceivable. Additionally, with a specific end goal to diminish the misuse of vitality green administration level assertion [3] is connected. The issue of vitality mindful 
dynamic situation of utilizations in a virtualized heterogeneous framework has communicated by Verma et al. [6] in light of keeping up the improvement. To accomplish this reason, the situation of virtual machines is advanced at each time allotment to diminish vitality use and increment execution. Analysts utilizing variable canister size and expenses to actualize a heuristic strategy for the receptacle pressing issue. In addition, the creators connected live movement of virtual machines in their investigation while the prescribed calculations don't think about the correct prerequisites of SLA. Kusic et al. The primary objective of this examination was to expand the advantages of the asset supplier by decreasing vitality use and SLA shock. Also, to estimate the measure of future requests, anticipating the up and coming condition of the framework, and performing basic re-appointment Kalman filteris utilized.

For allotting the assets in an approach to keep the data transfer capacity guarantees, Guo et al. [4] in a paper prescribed and played out a virtual group administration strategy. The distribution is determined through a heuristic technique that lessens the entire transfer speed utilized. Designating the virtual machine is balanced when a portion of the virtual machines are deallocated. In addition, designating the VM isn't balanced in view of the present system stack powerfully. Be that as it may, this strategy does not clearly decrease vitality utilization by the system. The recommended technique thought a national travel site as a genuine contextual analysis for investigates. The creators blended two noteworthy procedures: 1) memory pressure and 2) ask for separation. The memory pressure changes vitality devoured by CPU into memory ability to allow more errands combination. The ask for segregation blockaded unneeded solicitations to evacuate unfriendly asset utilization. The results of this investigation demonstrate the effectiveness of the proposed procedure since the assignment was performed with $20 \%$ less computations. Subrata et al. [3] displayed a vitality mindful booking model for web-scale frameworks which comprises of Nash Bargaining Solution. Also, this model for decreasing vitality utilization, maintains a stamped QoS administer among all the specialist organizations. The level of vitality utilization controlled a particular limit limitation to keep the proper QoS measure was resolved through SLA.

Mazzucco et al. [4] suggested a power mindful asset designation method to build the advantages procured through specialist co-ops. The asset allotment procedures presented in his examination depend on and off. The primary objective of this exploration was to diminish the measure of vitality utilization by improving the utilization of the servers. Fujiwara et al. [5] displayed a market based model to dispense different asset asks for in a Cloud Computing condition. This system makes the assets virtual and conveys them to the end-clients as different arrangement of administrations. The calculation proposed in this investigation expanded figuring from the specialist co-ops as an utility. The experimental results display that the suggested approaches increment the likelihood of apportioning assets in distributed computing frameworks. EnaCloud is another approach proposed by $\mathrm{B} \mathrm{Li}$ et al. [6]. Concerning the vitality productivity in distributed computing stages, this technique progressively empowers application live arrangement. EnaCloud utilized a VM to embody the demand that gives live movement and applications planning to lessen the quantity of dynamic machines as to scale up the vitality effectiveness. A vitality mindful exact calculation is prescribed to get a reasonable arrangement, for receptacle pressing issue in the application situation.

Liu et al. [7] shows the Green Cloud engineering to diminish server farm control utilization amid certification of the usage from the point of view of the clients. Green Cloud design engages live virtual machine movement, broad web based checking and 
virtual machine arrangement streamlining. To authenticate the viability and proficiency of committed design, analysts utilize a genuine internet amusement as a VM application called Tremulous. The aftereffects of assessment demonstrate that it is conceivable to store $27 \%$ of the vitality when utilizing Green Cloud engineering. Lee et al. [8] demonstrates two vitality mindful assignment combination heuristics that plan to expand asset effectiveness and certainly to contemplate both sit out of gear and dynamic vitality utilization. This examination distributes each errand to the asset in which the vitality utilization for performing.

\section{Existing power competent methods concentrating on network}

The exploration group has been distinguished system framework as another essential vitality purchaser asset. Gupta et al. [5] exhibits that putting inactive connections, switches and switches into rest modes in a system can decrease vitality utilization and spare the vitality use in Internet foundations. Numerous scientists considered on the vitality effectiveness in rush hour gridlock steering and performing rest modes and execution scaling of system parts, as indicated by the assessment expressed by Gupta et al. [5].

So as to progressively dedicate a gathering of dynamic system hubs to serve activity heap of server farm, Heller et al. [5] recommends ElasticTree, an energyaware arrange enhancer. ElasticTree chooses the gathering of system hubs which should stay dynamic to pick up the execution of the system that, later on, diminishes the greatest number of pointless witches and hubs as it can. Creators apply voracious canister packer strategy, expectation techniques, and topology-mindful heuristic toward determining the fitting system segments backup.

Results of reproduction exhibit that sparing around half of vitality in server farm occurs by ElasticTree. Si et al. [4] presents a vitality mindful distributed plan, eAware, to spare vitality by exploring the use of switch ports and latent system parts. The essential objective of this approach is depending on the line lengths. While a line length of a port is more prominent than specific limit different ports will be empower to decrease the line. In the other hand, if there isn't any errand in a port, it will be impaired. If all ports of a switch are workless, turn will be turned off. The consequences of recreation showed that eAware can ensure 30\%-half of vitality in server farm. Botero et al. [2] in a paper demonstrates the power mindful virtual system installing issue. The specialists apportioned the gathering of virtual systems to the diminished gathering of physical system parts and killing the sit switches and connects. Creators propose Mixed Integer Program [5] to settle the power mindful virtual system inserting issue. The outcomes demonstrate that the suggested approach diminishes the vitality utilization up to $35 \%$ when the activity low. In another investigation, Xu et al. builds up a throughput-ensured vitality mindful steering calculation. The rule objective of this investigation is to utilize the base system vitality to set up the steering administration, while thinking about the decided system throughput.

Prescribed steering calculation appraises the courses for the activity streams and system throughput and after that takes out connections and switches since the system throughput meets the minor limit. In the long run sit still connections and switches are changed to rest mode. Reproduction comes about recommend that the offered directing calculation in this exploration can diminish vitality utilization in server farms while arrange workload is low. Chiaraviglio and Matta [7] in an investigation present coordinated effort between Internet specialist organizations (ISPs) and segment suppliers 
that empowers the accomplishment of a compelling simultaneous distribution of system ways and process assets to decrease vitality use with respect to keep the execution of system. In another investigation, Tomas et al. [8] considers the issue of booking Message Passing Interface (MPI) undertakings with respect to keep the nature of administration necessities while information moves in the system. Some different examinations have been done on the warm proficiency of asset administration in server farms condition [9]. The investigates outlined that additional power funds is accomplished by temperature mindful workload area and the product based warm administration.

\section{Existing power resourceful methods concentrating on server and network}

This strategy actualizes activity union and VM to spare vitality in server farm systems. In any case, repetition prerequisites counteract killing inert hubs and virtual machine position in organize. In this way, Honeyguide proposed as an answer for sidestep connects to scale up the measure of system switches. Along these lines button can be killed under the terms of repetition. Honeyguide is actualized for fattree topology and utilizes the primary fit calculation to find VM. VMs are isolated into a progression of gatherings where general activity between the gatherings is limited while general movement of inside gathering is expanded. System activity aggregated into fewer courses to put the remaining system segments into rest mode for lessening vitality utilization. Virtual Machine Planner (VMP) is evaluated in a genuine server farm. The consequence of recreation introduces that VMP can spare a lot of vitality.

An engineering system was produced by Beloglazov et al. [3] for sparing vitality in dim compute. In light of this examination, specialists offered vitality mindful distribution heuristics arrangement for server farm parts to expand vitality sparing in server farms. This examination has assessed the vitality mindful asset designation calculations utilizing the solidification of Virtual Machines powerfully. The aftereffects of this paper show that this strategy significantly lessens vitality utilization in cloud server farms. In an investigation, Kliazovich et al. [16] reenacts vitality mindful for distributed computing server farms. Specialists planned this test system to get the data about vitality devoured through server farm components like servers, switches, and interfaces. The aftereffects of reenactment decided the viability of utilizing power administration composition like shutdown arranges components powerfully, recurrence scaling and voltage scaling [16]. The issue of energy productive asset administration in heterogeneous condition in Internet facilitating focuses is contemplated by Chase et al. For serving solicitation to each administration, the proposed approach chooses a dynamic arrangement of servers. Keeping in mind the end goal to change the dynamic arrangement of servers, the system switches are reconfigured progressively as required. By putting latent servers into control sparing mode, similar to rest and hibernation, vitality use is diminished.

In another investigation, the issue of dealing with the vitality proficiency of assets in a web-application condition has investigated by Elnozahy et al.. The creators utilized DVFS and changing the energy of processing hubs on/off as the vitality sparing strategies in their examination. The fundamental thought behind this examination is to indicate ideal measure of physical hubs, modify the suitable recurrence to every one of the hubs and to assess the aggregate recurrence of CPU basic to display the required reaction time. As indicated by optical burst changing innovation to limit undertaking consummation times, Koseoglu and Karasan have applied a comparative technique for joining distribution of system ways and computational assets in Grid situations. Gyarmati and Trinh look at the results of vitality utilization of system models in server farms. System structures 
enhancement can be actualize at configuration time of server farm and can't be perform progressively. Creators expressed that the vitality utilization of the server farm models show distinctive credits correspondingly to the capacity of transmission. The recreation aftereffects of their investigation show that there is an exchange off between vitality requirements and the broadcast abilities. Gill et al. [15], demonstrated a recreation domain for server farm of vitality mindful distributed computing. Close to the workload scattering, the test system is intended to get subtle elements of the vitality that is utilized by server farm segments notwithstanding pocket level correspondence display between them. The aftereffects of recreation picked up for 2-level, 3-level and 3-tire with rapid server farm structures that play out the test system adequacy in utilizing different power organization pattern, similar to dynamic shutdown, recurrence scaling and voltage scaling.

\section{Conclusion}

The paper begins by presenting the idea of distributed computing, green registering and afterward the different procedures by which Information innovation is moving towards Green IT. A proficient and successful utilization of processing assets in cloud influence it To green Cloud registering, Some methodologies examined in this paper utilize workload designation and planning and disjoin profiling without utilization of virtualization others make utilization of virtualization system. Decreasing carbon discharge and vitality utilization in distributed computing server farms make an open test and arrange toward making green server farms. A viable and effective utilization of figuring assets in cloud can help in accomplishing Green Cloud Computing. The connected investigate proposition is generally centered on vitality sparing methodologies for server farms. Be that as it may, because of expanding request on transmission capacity and system availability of server farm, vitality utilization of server farm system and server farm servers and system will quickly develop later on. This paper introduces the vast majority of the innovations utilized for vitality sparing in server farms and examines different methodologies proposed in past research works in this field. The investigation uncovers that there are numerous vitality proficient structures for distributed computing and server farms that make distributed computing a Green distributed computing.

\section{References}

[1] J. Liu, F. Zhao, X. Liu, and W. He, "Challenges towards elastic power management in internet data centers," in Distributed Computing Systems Workshops, 2009. ICDCS Workshops' 09. 29th IEEE International Conference on 2009, pp.65-72, (2009)

[2] G. Chen, W. He, J. Liu, S. Nath, L. Rigas, L. Xiao, et al., "Energy-Aware Server Provisioning and Load Dispatching for Connection-Intensive Internet Services," in NSDI, pp.337-350, (2008)

[3] J. Chabarek, J. Sommers, P. Barford, C. Estan, D. Tsiang, and S. Wright, "Power awareness in network design and routing," in INFOCOM 2008. The 27th Conference on Computer Communications. IEEE, (2008).

[4] C. Gunaratne, K. Christensen, B. Nordman, and S. Suen, "Reducing the energy consumption of Ethernet with adaptive link rate (ALR)," Computers, IEEE Transactions on, vol.57, pp.448-461, (2008)

[5] J. F. Botero, X. Hesselbach, M. Duelli, D. Schlosser, A. Fischer, and H. De Meer, "Energy efficient virtual network embedding," Communications Letters, IEEE, vol.16, pp.756-759, (2012)

[6] A. Fischer, J. F. Botero, M. Till Beck, H. De Meer, and X. Hesselbach, "Virtual network embedding: A survey," Communications Surveys \& Tutorials, IEEE, vol.15, pp.1888- 1906, (2013)

[7] Y. Shang, D. Li, and M. Xu, "Energy-aware routing in data center network," in Proceedings of the first ACM SIGCOMM workshop on Green networking, pp.1-8, (2010) 
[8] P. Mahadevan, P. Sharma, S. Banerjee, and P. Ranganathan, "Energy aware network operations," in INFOCOM Workshops 2009, IEEE, pp.1-6, (2009)

[9] Apple. Apple Facilities Environmental Footprint Report. Available: http://www.apple.com/environment /reports/do cs/Apple_Facilities_Report_2013.pdf, (2012)

[10] Gandhi, M. Harchol-Balter, R. Das, and C. Lefurgy, "Optimal power allocation in server farms," in ACM SIGMETRICS Performance Evaluation Review, pp.157-168, (2009)

[11] R. V. Aroca and L. M. G. Goncalves, "Towards green data centers: A comparison of x86 and ARM architectures power efficiency," Journal of Parallel and Distributed Computing, vol.72, pp.1770- 1780, (2012)

[12] C. Guo, G. Lu, H. J. Wang, S. Yang, C. Kong, P. Sun, et al., "Secondnet: a data center network virtualization architecture with bandwidth guarantees," in Proceedings of the 6th International COnference, pp.15, (2010)

[13] J. Torres, D. Carrera, K. Hogan, R. Gavalda, V. Beltran, and N. Poggi, "Reducing wasted resources to help achieve green data centers," in Parallel and Distributed Processing, IPDPS 2008. IEEE International Symposium on 2008, pp.1-8, (2008)

[14] R. Subrata, A. Y. Zomaya, and B. Landfeldt, "Cooperative power-aware scheduling in grid computing environments," Journal of Parallel and Distributed Computing, vol.70, pp.84-91, (2010)

[15] M. Mazzucco, D. Dyachuk, and R. Deters, "Maximizing cloud providers' revenues via energy aware allocation policies," in Cloud Computing (CLOUD), 2010 IEEE 3rd International Conference on 2010, pp.131-138, (2010)

[16] I. Fujiwara, K. Aida, and I. Ono, "Marketbased Resource Allocation for Distributed Computing," vol.2009, pp.1-6, (2009)

[17] B. Li, J. Li, J. Huai, T. Wo, Q. Li, and L. Zhong, "Enacloud: An energy-saving application live placement approach for cloud computing environments," in Cloud Computing, 2009, CLOUD’09. IEEE International Conference on 2009, pp.17-24, (2009)

[18] L. Liu, H. Wang, X. Liu, X. Jin, W. B. He, Q. B. Wang, et al., "GreenCloud: a new architecture for green data center," in Proceedings of the 6th international conference industry session on Autonomic computing and communications industry session, pp.29-38, (2009) 
Green Cloud Computing: Some Studies on Energy Usage Reduction Methods

This page is empty by intention. 